\title{
Assessing Rural Sustainable Development Potentialities using a Dominance-based Rough Set Approach
}

Antonio Boggia*, University of Perugia Borgo XX Giugno, 7406121 Perugia Italy. Email: antonio.boggia@unipg.it Lucia Rocchi, University of Perugia Borgo XX Giugno, 7406121 Perugia Italy. Email: lucia.rocchi@unipg.it Luisa Paolotti, University of Perugia Borgo XX Giugno, 7406121 Perugia Italy. Email: luisa.paolotti@gmail.com Francesco Musotti, University of Perugia Borgo XX Giugno, 7406121 Perugia Italy. Email: francesco.musotti@unipg.it

Salvatore Greco, University of Catania, Corso Italia, 5595129 Catania Italy and Portsmouth Business School, Operations \& Systems Management University of Portsmouth, Portsmouth PO1 3DE, United Kingdom. Email: salgreco@unict.it

*Corresponding author

Antonio Boggia

University of Perugia

Borgo XX Giugno, 74

06121 Perugia Italy

phone: +39075 5857136

fax: +39075 5857146

e-mail: antonio.boggia@unipg.it 


\title{
Assessing Rural Sustainable Development Potentialities using a Dominance-based Rough Set
} Approach

\begin{abstract}
Rural Development is a priority in Europe and it is supported by specific, financial programmes. At the same time, sustainability is the key word for the European Union to construct programmes and policies for all human activities. However, measuring sustainability of rural areas is not easy, due to their particular features. The improvement of knowledge on sustainability in rural areas is important to build long term policies and strategies for those territories. The objective of this study is the development of a decision support system based on the Dominance-based Rough Set Approach (DRSA), to assess the level of Rural Sustainable Development in specific areas. We used DRSA to analyze the level of sustainability of the 92 municipalities of the Region of Umbria, Italy. The results were synthesized in a final ranking, taking into account the equilibrium and the integration between development and sustainability of each municipality. DRSA showed a high potential in the context of management or planning, and for supporting Decision Makers. DRSA is able to give a ranking as well as an explanation of the main factors driving sustainable development in rural areas.
\end{abstract}

Keywords: Rural development, Sustainable development, Dominance-based Rough Set Approach, Multi-criteria analysis.

\section{Introduction}

Rural areas in Europe represent a large part of the territory. Approximately $86 \%$ of the territory and approximately $75 \%$ of the LAU2 units of the European Union are rural (Jonard et al., 2009; United Nation, 2008). LAU stands for Local Administrative Unit corresponding to the lowest level administrative of a country. In the European Union, LAUs form the basic element of the Nomenclature of Territorial Units for Statistics (NUTS) of the regions. LAU2 represents the level of municipalities, or similar. LAU1 is an upper level, which is not defined in all UE countries. They present very different environments, a variety of economic activities, unique and ancient, social and cultural traditions (Heilig, 2002). Rural development is the second pillar of the Common Agricultural Policy, and financial aids are available for the development of rural regions and communities in the EU (Gómez-Limón and Atance, 2004). Furthermore, the agricultural and rural policy of EU has increased the attention paid to the environment in the last ten years (Young et al., 2005; Hart and Baldock, 2011). Sustainability of human activities is one of the most important concerns of the European Union. Sustainable development was introduced for the first time as an explicit recommendation in the Brundtland report of 1987. A requirement for environmental integration in all policies was introduced by the Maastricht Treaty in 1992 and reinforced by the Treaty of Amsterdam (1997). Europe 2020 (COM (2010) 2020), the EU's growth strategy for the coming decade, is the final act in this direction. However, the discussions on what the strategic objectives of sustainable agriculture are, which criteria are to be taken into account, which are the actions to develop, and which are the methodological tools to use for the involved evaluations, are 
still under development (Young et al., 2005). As a consequence, the need to assess the level of sustainability achieved, both at company and at government level, is increasing. This process involves all economic sectors, including agriculture. In 1988, the Food and Agriculture Organization (FAO) Council defined sustainable development as "the management and conservation of the natural resource and the orientation of technological and institutional change in such a manner as to ensure the attainment and continued satisfaction of human needs for present and future generations. Such sustainable development in the agriculture, forestry and fisheries sectors conserves land, water, plant and animal genetic resources, is environmentally non-degrading, technically appropriate, economically viable and socially acceptable" (FAO, 1988).

In rural areas, development must be achieved to improve social and economic conditions, but it must be sustainable, following the guide lines drawn in the Renewed EU Sustainable Development Strategy, published in 2006 and 2009. Rural development must become a Rural Sustainable Development (Copus and Crabtree, 1996).

The goals of rural sustainable development are (Baldock et al., 2001):

- diversification of agricultural production (Hjalager, 1999);

- multi-functionality of agriculture (enhance social, environmental and cultural functions of agriculture, in addition to the productive function) (Morgan et al., 2010; Wilson, 2007);

- enhancement of food security (Ayres and McCalla, 1996);

- employment and income generation in rural areas (Shortall and Shucksmith, 2001);

- natural resource management and environmental protection (Emerson and Gillmor, 1999);

- conservation of social and cultural traditions in rural areas (Kneafsey, 2001; Szlanyinka, 2009).

The result should be to enable rural communities to achieve the following goals:

- to be cohesive and stable with viable institutions and sustainable economies;

- to be able to attract and retain skilled people, capable of contributing to growth and development (Shortall and Shucksmith, 2001);

- to guarantee proper environmental management.

Rural sustainable development is a multidimensional concept (Kitchen and Marsden, 2009). It means that Multiple Criteria Decision Aiding (MCDA) (see Figueira et al., 2005 for a state of the art) is the most suitable tool for an assessment process. The Rural Development Programme in Europe is subjected to a constant evaluation and revision, as part of the Common Agriculture Policy (CAP). The same happens in other countries. For a better application of programmes and plans regarding rural development, it is very important to analyze and understand local opportunities and territorial characteristics. In this way, it is possible to guide and manage rural development. Assessing the level of Rural Sustainable Development in specific areas, and finding explanations for the different levels is important to address decision makers in their allocation of resources. This can help to maintain and improve both the level of development and the level of sustainability in rural areas.

The objective of this study is to develop a decision support system based on the Dominancebased Rough Set Approach (DRSA) (Greco et al., 2001a), to assess the level of Rural Sustainable 
Development in specific areas, and to address decision makers in the allocation of their resources, to maintain and improve the level of rural sustainable development.

The case study refers to Umbria, a region of central Italy. A set of indicators of rural development and sustainable development was determined. This set reflects rural sustainable development at a territorial level (in this case at municipality level). The analysis considers the 92 municipalities of Umbria.

The paper is structured as follows: after an accurate overview of the DRSA method in Section 2 , the indicators used in the analysis and the case study are described in Section 3 . Section 4 reports the principal results, whereas Section 5 reports the discussion. Some final conclusions follow.

\section{Method}

The Rough Sets theory was introduced by Pawlak (Pawlak, 1982, 1991) and it constitutes a tool to describe a set of objects, for which the available information is possibly inconsistent or ambiguous. The Rough Sets philosophy is based on the assumption that a certain amount of information (data, knowledge, etc.), expressed by means of certain attributes, is associated with every object of a universe $U$.

Rough sets are formally defined on the basis of an indiscernibility relation in a given universe of the discourse $U$, defined as follows: two objects from $U$ are indiscernible if they have the same description with reference to the available information. The indiscernibility relation is an equivalence relation and consequently, it allows a partition of the universe of objects under examination to be made. In this sense, it is possible to identify blocks of indiscernible objects, called elementary sets or granules, which can be used to build up new knowledge.

An information system is the basis on which the rough set theory is applied. It may contain two classes of attributes, known as condition and decision attributes, respectively, which may be used to transform the information system into a decision table. The decision table can then be denoted as $S=(U, C, D)$ where $C$ and $D$ are the condition and decision attribute sets, respectively. The decision attributes, together with the indiscernibility relation, are used to partition the information table into decision classes. The lower approximation of a given decision class consists of all the objects such that all objects indiscernible with them with respect to condition attributes are contained in the considered decision class. The upper approximation of a given decision class consists of all the objects for which there is at least one object indiscernible with them contained in the considered decision class. The object belonging to the upper approximation, but not belonging to the lower approximation constitutes the boundary of the considered decision class. Lower approximation, upper approximation and boundary represent the certain, possible and doubtful knowledge of the decision class, respectively.

To apply the rough set theory to MCDA, we need to generalize the Classical Rough Set Approach (CRSA) and consider preferences for attribute domains and for the set of decision classes. A rough set applied to MCDA also enables decisions to be considered which are "inconsistent" due to the limited discriminatory power of the criteria for the analysis or as a result of the hesitation of the Decision Maker (DM). Thus, in order to take into account the preferences of the DM and the inconsistency typical of decision problems, (Greco et al., 1999, 2001a, 2002) proposed an 
extension of the CRSA. This innovation is based on the substitution of the indiscernibility relation by a dominance relation in the rough approximation of decision classes, to obtain a new rough set model called the Dominance Rough Set Approach (DRSA). For some recent surveys on DRSA see Greco et al., 2005; Slowinski et al., 2009, 2012, 2014. Recently, the DRSA has also been applied to multi-objective optimization (Greco et al., 2008).

DRSA considers preference evaluations on the decision attributes and preference ordered classes defined by the decision attributes. Within DRSA the lower approximation of a set of objects belonging to at least a given class (i.e. the set of objects belonging to the given decision class or to a better decision class) consists of all the objects such that all objects being at least as good as them with respect to all condition attributes (i.e. all other objects dominating them) are contained in the considered decision class or in a better decision class. The upper approximation of a set of objects belonging to at least a given decision class consists of all the objects for which there is at least one object being at most as good as them with respect to all condition attributes (i.e. at least one object dominated by them) which is contained in the considered decision class or in a better decision class. Analogously, within DRSA the lower approximation of a set of objects belonging to at most a given decision class (i.e. the set of objects belonging to the given decision class or to a worse decision class) consists of all the objects such that all objects being at most as good as them with respect to all condition attributes (i.e. all other objects dominated by them) are contained in the considered decision class or in a worse decision class. The upper approximation of a set of objects belonging to at most a given decision class consists of all the objects for which there is at least one object being at least as good as them with respect to all condition attributes (i.e. at least one object dominating them) which is contained in the considered decision class or in a worse decision class. The CRSA, as already seen, is based on an equivalence relation, through which it is impossible to take the information related to preference ordered information into proper account. According to this theory, it is impossible to identify the inconsistency, due to the presence of ordered criteria among the attributes. In the DRSA, the set of decision rules induced gives a more synthetic representation of knowledge contained in the decision table, because the minimal sets of rules thus obtained have a smaller number of rules and use a smaller number of conditions. The reason is the difference between the set of rules induced from a classical approach and a set of rules coming from the DRSA. In fact, the application to new objects of DRSA rules expressed in the form "if... at least/at most..., then..." gives better results than the application of the CRSA rules expressed in the form "if... equal..., then...". The separation of certain and doubtful knowledge about the DM's preferences obtained by DRSA is expressed in terms of different kinds of decision rules, depending on whether they are induced from lower approximations of decision classes, or from the boundaries of these classes consisting of inconsistent examples that fail to observe the dominance principle.

Let us recall the basic concepts of the Dominance-based Rough Set Approach (DRSA).

The sets to be approximated are called upward unions and downward unions of classes, respectively:

$$
C l_{t}^{\geq}=\bigcup_{s \geq t} C l_{s}, \quad C l_{t}^{\leq}=\bigcup_{s \leq t} C l_{s}, \mathrm{t}=1, \ldots, \mathrm{n} .
$$


The statement $C l_{t}^{\geq}=\bigcup_{s \geq t} C l_{s}$ means "x belongs to at least class $\mathrm{Cl}_{\mathrm{t}}$ ", whereas $C l_{t}^{\leq}=\bigcup_{s \leq t} C l_{s}$ means "x belongs to class $\mathrm{Cl}_{\mathrm{t}}{ }^{\prime \prime}$ at most.

Let us observe that that $C l_{1}^{\geq}=C l_{n}^{\leq}=\mathrm{U}, C l_{n}^{2}=\mathrm{Cl}_{\mathrm{n}}$ and $\mathrm{Cl}_{1}^{\leq}=\mathrm{Cl}_{1}$. Furthermore, for $\mathrm{t}=2, \ldots, \mathrm{n}$, we have:

$$
C l_{t-1}^{\leq}=U-C l_{t}^{\geq} \text {and } C l_{t}^{\geq}=U-C l_{t-1}^{\leq} .
$$

Given $x$ and $y$ two objects in the universe, we say that object $x$ dominates object $y, x, y \in U$, with respect to $P \subseteq C$, denoted by $x D p y$, if $x$ is at least as good as $y$ with respect to all $q \in P$. Given $P \subseteq C$ and $x \in U$, the "granules of knowledge" used for approximation in DRSA are:

- a set of objects dominating $x$ with respect to criteria from $\mathrm{P}$, called the $\mathrm{P}$-dominating set, $D_{P}^{+}(x)=\{\mathrm{y} \in \mathrm{U}: \mathrm{yD} \mathrm{pX}\}$

- a set of objects dominated by $\mathrm{x}$ with respect to criteria from $\mathrm{P}$, called the P-dominated set, $D_{P}^{-}(x)=\{\mathrm{y} \in \mathrm{U}: \mathrm{xD} \mathrm{p} y$.

For any $\mathrm{P} \subseteq \mathrm{C}$, we say that $\mathrm{x} \in \mathrm{U}$ belongs to $C l_{t}^{\geq}$without any ambiguity if $\mathrm{x} \in \mathrm{Cl}_{t}^{\geq}$and, for all the objects $\mathrm{y} \in \mathrm{U}$ dominating $\mathrm{x}$ with respect to $\mathrm{P}$, we have $\mathrm{y} \in C l_{t}^{\geq}$, i.e. $D_{P}^{+}(x) \subseteq C l_{t}^{\geq}$. Furthermore, we say that $\mathrm{y} \in \mathrm{U}$ could belong to $C l_{t}^{\geq}$if there at least one object $\mathrm{x} \in C l_{t}^{\geq}$were to exist, such that $\mathrm{y}$ dominates $\mathrm{x}$ with respect to $\mathrm{P}$, i.e. $\mathrm{y} \in D_{P}^{+}(x)$.

Thus, with respect to $\mathrm{P} \subseteq \mathrm{C}$, the set of all objects belonging to $C l_{t}^{\geq}$without any ambiguity constitutes the P-lower approximation of $\mathrm{Cl}_{t}^{\geq}$, denoted by $\underline{P}\left(\mathrm{Cl}_{t}^{\geq}\right)$, and the set of all objects that could belong to $C l_{t}^{\geq}$constitutes the P-upper approximation of $C l_{t}^{\geq}$, denoted by $\bar{P}\left(C l_{t}^{\geq}\right)$:

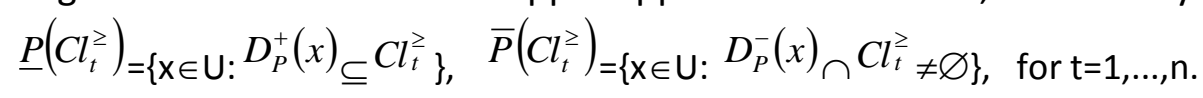

Analogously, using $D_{P}^{-}(x)$, we can define P-lower approximation and P-upper approximation of $C l_{t}^{\leq}$:

$$
\left.\underline{P}\left(C l_{t}^{\leq}\right)=\left\{\mathrm{x} \in \mathrm{U}: D_{P}^{-}(x)_{\subseteq} C l_{t}^{\leq}\right\}, \quad \bar{P}\left(C l_{t}^{\leq}\right)_{=\{\mathrm{x} \in \mathrm{U}:} D_{P}^{+}(x)_{\cap} C l_{t}^{\leq} \neq \varnothing\right\}, \text { for } \mathrm{t}=1, \ldots, \mathrm{n} .
$$

The P-boundaries (P-doubtful regions) of $\mathrm{Cl}_{t}^{\geq}$and $\mathrm{Cl}_{t}^{\leq}$are defined as:

$$
\left.\mathrm{BnP}\left(C l_{t}^{\geq}\right)=\bar{P}\left(C l_{t}^{\geq}\right)_{-}-\underline{P}\left(C l_{t}^{\geq}\right), \quad \mathrm{BnP} C l_{t}^{\leq}\right)=\bar{P}\left(C l_{t}^{\leq}\right)_{-} \underline{P}\left(C l_{t}^{\leq}\right), \text {for } \mathrm{t}=1, \ldots, \mathrm{n} \text {. }
$$

For a given upward or downward union of classes, $\mathrm{Cl}_{t}^{\geq}$or $\mathrm{Cl}_{s}^{\leq}$, the decision rules induced under a hypothesis that objects belonging to $\underline{P}\left(\mathrm{Cl}_{t}^{\geq}\right)$or $\underline{P}\left(\mathrm{Cl}_{s}^{\leq}\right)$are positive and all the others negative, suggest a certain assignment to "at least class $\mathrm{Cl}_{t}$ " or to "at most class $\mathrm{Cl}_{s}$ ", respectively; on the other hand, the decision rules induced under a hypothesis that objects belonging to the intersection $\bar{P}\left(\mathrm{Cl}_{s}^{\leq}\right) \cap \bar{P}\left(\mathrm{Cl}_{t}^{\geq}\right)$are positive and all the others negative, suggest an approximate assignment to some classes between $\mathrm{Cl}_{s}$ and $\mathrm{Cl}_{\mathrm{t}}(\mathrm{s}<\mathrm{t})$.

Assuming that, for each $q \in C, V_{q} \subseteq R$ (i.e. $V_{q}$ is quantitative) and that, for each $x, y \in U, f(x, q) \geq f(y, q)$ implies that $x$ is at least as good as $y$ with respect to criterion $q$, denoted by $x \succeq_{q} y$, the following two types of decision rules can be considered: 
1) $D \geq$-decision rules with the following syntax:

if $f\left(x, q_{1}\right) \geq r_{q 1}$ and $f\left(x, q_{2}\right) \geq r_{q 2}$ and $\ldots f\left(x, q_{p}\right) \geq r_{q p}$, then $x \in C l$,

where $P=\left\{q_{1}, \ldots, q_{p}\right\} \subseteq C,\left(r_{q 1}, \ldots, r_{q p}\right) \in V_{q 1} \times V_{q 2} \times \ldots \times V_{q p}$ and $t \in\{2, \ldots, n\}$;

2) $D \leq$-decision rules with the following syntax:

if $f\left(x, q_{1}\right) \leq r_{q 1}$ and $f\left(x, q_{2}\right) \leq r_{q 2}$ and $\ldots f\left(x, q_{p}\right) \leq r_{q p}$, then $x \in C l$,

where $P=\left\{q_{1}, \ldots, q_{p}\right\} \subseteq C,\left(r_{q 1}, \ldots, r_{q p}\right) \in V_{q 1} \times V_{q 2} \times \ldots \times V_{q p}$ and $t \in\{1, \ldots, n-1\}$.

Sometimes probabilistic decision rule are considered (Greco et al., 2001b, Blaszczynski et al., 2009). In this case each rules has a credibility measured as the ratio between the number of objects satisfying the premise and the conclusion over the number of objects satisfying only the premise, i.e.

1) in case of a $D \geq$-decision rule $r$ with the following syntax:

if $f\left(x, q_{1}\right) \geq r_{q 1}$ and $f\left(x, q_{2}\right) \geq r_{q 2}$ and $\ldots f\left(x, q_{p}\right) \geq r_{q p}$, then $x \in C l$, the credibility $C(r)$ of the rule $r$ is given by

$$
C(r)=\frac{\operatorname{card}\left(x \in U: f\left(x, q_{1}\right) \geq r_{q 1} \text { and } f\left(x, q_{2}\right) \geq r_{q 2} \text { and } \ldots f\left(x, q_{p}\right) \geq r_{q p} \text { and } x \in C l_{t}^{\geq}\right)}{\operatorname{card}\left(x \in U: f\left(x, q_{1}\right) \geq r_{q 1} \text { and } f\left(x, q_{2}\right) \geq r_{q 2} \text { and } \ldots f\left(x, q_{p}\right) \geq r_{q p}\right)} ;
$$

2 ) in case of a $D \leq$-decision rule $r$ with the following syntax:

if $f\left(x, q_{1}\right) \leq r_{q 1}$ and $f\left(x, q_{2}\right) \leq r_{q 2}$ and $\ldots f\left(x, q_{p}\right) \leq r_{q p}$, then $x \in C l_{t}^{\leq}$, the credibility $C(r)$ of the rule $r$ is given by

$$
C(r)=\frac{\operatorname{card}\left(x \in U: f\left(x, q_{1}\right) \leq r_{q 1} \text { and } f\left(x, q_{2}\right) \leq r_{q 2} \text { and } \ldots f\left(x, q_{p}\right) \leq r_{q p} \text { and } x \in C l_{t}^{\leq}\right)}{\operatorname{card}\left(x \in U: f\left(x, q_{1}\right) \leq r_{q 1} \text { and } f\left(x, q_{2}\right) \leq r_{q 2} \text { and } \ldots f\left(x, q_{p}\right) \geq r_{q p}\right)} \text {. }
$$

\section{Case study}

\subsection{Indicators}

The phenomenon of rurality is extremely complex to focus on, and to translate into empirical measurement, as a consequence of its multidimensional qualities (social, geographical, demographical, economic, etc.), and of its variability in time. This complexity makes it quite hard to give a precise definition of rurality.

The choice of any definition is always partial and susceptible to absolutely legitimate criticism (Murdoch and Pratt, 1997). Even the definitions adopted for some years by OECD (OECD, 1994, 1996) and EU (European Commission, 1997) should nowadays be subjected to serious discussion. The OECD definition is too centred on demographic density and on geo-statistical units, giving it an administrative nature instead of a functional one (socially and economically intended). On the other hand, the EU definition is ambiguously centred on the idea of economic backwardness (a rural area as being synonymous with a lagging area). Both definitions stem from a dual approach to economic-territorial analysis (rural vs. non rural) instead of a more realistic standpoint, i.e. the consideration of a plurality of local development patterns, with a plurality of rural local models as well. 
Since we are unable to discuss this theory more in depth in the present paper, we use the explicitly "empirical and reductive" definition of rurality given by Kaiser (Kaiser, 1990), founded on the following four general points:

1. low density of inhabitants and buildings and therefore prevalence of green landscapes;

2. prevalently agricultural, forest and pastoral use of space;

3. inhabitants' lifestyle characterized by belonging to small centres and a very close and penetrating relationship with the environment;

4. specific identity and self-representation of people influenced by a peasant background.

Based on census data (ISTAT) referring to 2010 (for agriculture) and 2011 (on population) (ISTAT, 2010, 2011), we pointed out some criteria that seem to represent a reasonable translation into quantitative benchmarks of Kaiser's points. Quantitative benchmarks must be intended in relative terms rather than in absolute terms: more rural (or less urban) versus less rural (or more urban), instead of a clear contrast between urban and rural. Moreover, the criteria analyzed are coherent with the socio-economic dimension of suitable development for the rural area (Copus and Crabtree, 1996)

These five criteria are as follows:

1. the quota of freehold dwellings (that is generally higher in the small settlements of rural localities);

2. the quota of the population resident in minor centres (centres with a number of residents not exceeding the number of the most populated cluster of houses in the region of Umbria (Istat, 2011), clusters of houses and scattered houses (as a proxy of a typical settlement in rural localities);

3. the demographic density, measured according to the residents in major centres, that is centres with a number of residents exceeding the number of the most populated cluster of houses in region (measure of the absolute centralization of settlement: the greater it is, the greater the phenomena of both residential and productive social gravitation);

4. the number of residents (measure of the scale of settlement: the greater it is, the greater the phenomena of both residential and productive agglomeration);

5. the ratio between young farmers (under 40 years old) and the rest of employers in the primary sector (that measure the turnover in a key sector for rural areas).

Moreover, two indicators were used to give more information concerning the level of rural development. The two indicators relate to the capacity to supply products and services in addition to the traditional commodities: in this case typical products and provision of accommodation in rural tourism. Finally, three indicators relating to the level of irrigation, density of breeding and the level of organic agriculture were added to complete the framework.

The following is the complete list of the indicators used for the analysis:

A1: Population scattering index: this is the ratio between the population living in individual homes, villages, small towns (up to 518 units), and the total population.

A2: Concentrated population/Sq. Km: this is the concentrated population per unit of area (square $\mathrm{km}$ ).

A3: Owned homes/Total homes: this is the ratio between the number of homes owned by the residents and the total number of homes. 
A4: Total residents: this is the total number of residents.

A5: Agricultural age structure: this is the ratio between farms managed by young farmers and the total number of farms.

A6: Typical products rate: this is the ratio between the number of typical products from agriculture in the municipality and the total number of typical products of Umbria.

A7: No. of bed spaces in rural tourism accommodation/Sq.km: this is the number of bed spaces in rural tourism accommodation per unit of area (square $\mathrm{km}$ ).

A8: Irrigation water use/UAA: this is the amount of water used for irrigation per unit (Hectare) of Utilized Agricultural Area (UAA)

A9: Livestock standard units/UAA: this is the number of animals breeding converted into Livestock Standard Units per unit of UAA

A10: Organic UAA/UAA: this is the ratio between organic UAA and the total UAA.

Key subject indicators for sustainable rural development are required, but are difficult to apply to different contexts (Schultink, 2000). The above is a possible set, suitable for this case study, but of course more research and studies need to be developed.

\subsection{Assessing Rural Sustainable Development in Umbria, Italy}

We applied the analysis to the 92 municipalities of the Italian Region of Umbria (Figure 1).

\section{Insert Figure 1}

According to the OECD classification, all the municipalities in Umbria are predominantly rural or significantly rural: no predominant urban areas are to be found. In such a classification, the concept of sustainability is not taken into account.

Therefore, we calculated each indicator for the 92 municipalities. The level of rural sustainable development of each municipality was described by means of the 10 selected criteria. Table 1 shows a frame of the effects table: in the columns are the indicators, while in the rows Municipalities. In each cell the performance of each municipality with respect to the related indicator is reported.

Insert Table 1

After calculating the indicators, DRSA was applied. DRSA requires exemplary cases to be selected. For this purpose, we asked for an evaluation by a regional expert of the level of rural sustainable development (RSD) for 18 of the 92 municipalities, to be used as a point of reference. The 18 municipalities were classified into three groups: good level of RSD (Municipalities of Paciano, Lisciano Niccone, Preci, Sellano, Monteleone di Spoleto and Montefranco); average level of RSD (Municipalities of Ficulle, Spoleto, Gubbio, Trevi, Baschi and Cannara); poor level of RSD (Municipalities of Giove, Attigliano, Perugia, Bastia Umbra, Penna in Teverina and Terni). By using this classification, we induced the decision rules according to the Dominance-based Rough set Approach.

The next step was the application of the rules to all 92 municipalities. For each municipality we calculated:

- the rules matching each group of classes: good, at least average, mostly average, poor;

- the minimal credibility of assignment for each group of classes. 
The information given by the rules was synthesized into a total score $M$, calculated for each municipality, as follows:

$$
\text { Score }(M)=\operatorname{Score}^{+}(M)-\operatorname{Score}^{-}(M)
$$

where: $\operatorname{Score}^{+}(M)=$ sum of the credibility $C(r)$ of matched rules for which $M$ is at least average (i.e. good or average);

Score $(M)=$ sum of the credibility $C(r)$ of matched rules for which $M$ is at most average (i.e. poor or average).

This score is monotonic with respect to the evaluation of the considered criteria and agrees with the two principles that:

1. the more numerous and the more credible the positive rules (classifying the municipalities good or at least average) the greater the Score(M);

2. the more numerous and the more credible the negative rules (classifying the municipalities poor or at most average) the smaller the Score(M).

Thus, we obtained a ranking of the level of sustainable rural development of municipalities.

\section{Results}

The results of the application of DRSA to the 18 municipalities were as follows:

- 39 decision rules, describing good municipalities.

An example is: if the Population is $\leq 1208$ and the Organic UAA/UAA is $\geq 1.37 \%$, then the municipality is good (fits for the municipalities of Preci, Sellano and Monteleone di Spoleto).

- 76 decision rules describing at least average municipalities.

An example is: if the Population scattering index is $\geq 0.29$ and the Owned homes/Total homes are $\leq 0.75$, then the municipality is at least average (fits for the municipalities of Lisciano Niccone, Preci, Sellano, Spoleto and Cannara).

- 63 decision rules describing at most average municipalities.

An example is: if the Concentrated population/Sq. $\mathrm{Km}$ is $\geq 12.3115$ and the Agricultural employed residents/Total residents are $\leq 0.0434$, then the municipality is at most average (fits for the municipalities of Giove, Attigliano, Perugia, Bastia Umbra, Spoleto, Gubbio, Trevi, Baschi, Cannara).

- 42 decision rules describing poor municipalities.

An example is: if the Typical products rate is $\leq 1.12 \%$ and the No. of bed spaces in rural tourism accommodation/Sq.km is $\leq 1.086169$, then the municipality is poor (fits for the municipalities of Giove, Attigliano, Bastia Umbra, Penna in Teverina, Terni)

To give an idea of the contribution of each criterion to the development of the rules, Table 2 shows the presence of criteria in decision rules and their importance for a good, average or poor result. For example: the criterion Concentrated population/Sq $\mathrm{Km}$ is present only in 10 decision rules. Moreover, in 5 cases it is determinant for good or at most average results, while in other 5 cases for at least average or poor results. Therefore, the contribution to the best and to the worst results is the same. On the contrary, the criterion Livestock standard units/UAA, for instance, is present in 44 rules and has a greater importance for good and at most average results, than for an at least average or poor classification. 


\section{Insert Table 2}

The results of the application of the rules to all the 92 municipalities were synthesized, thanks to the calculation of Score(M), into a final ranking. The first ten municipalities and their response to rules are reported in Table 3.

The table shows the scores and the confidence of rules. For each upward and downward union of classes (good, at least average, at most average, poor) the number of rules and the sum of confidence is reported ("a" stands for the number of rules," $b$ " stands for the sum of confidence).

\section{Insert Table 3}

\section{Discussion}

What we tried to achieve with this application of DRSA was not merely a classification of territory. We wanted to evaluate the potentialities of the method for supporting the Decision Makers (DMs) in planning and managing rural areas. In particular, we wanted to evaluate, thanks to DRSA, the level of rural sustainable development in the municipalities.

The DRSA proved to be very effective in the assessment of sustainable rural development of the municipalities in Umbria. Moreover, the details of the information in the final results showed an important characteristic of DRSA: traceability. Traceability means that from the final score we can go back to the rules of reference, and from the rules to the objects in the data set.

For example, for the rule "if the No. of beds spaces in rural tourism accommodation/Sq.km is $\leq 2.00$, and the Organic UAA/UAA is $\leq 0.03$, then the municipality is poor in $75 \%$ of cases", the traceability says that this rule is supported by municipalities of Sellano, Montefranco, Giove, Attigliano, Perugia, Bastia Umbra, Penna in Teverina, Terni. Analogously, for to the rule "if the No. of bed spaces in rural tourism accommodation/Sq.km is $\leq 1.09$ and the Organic UAA/UAA is $\leq$ 0.01 , then the municipality is poor in $100 \%$ of cases", the traceability says that this rule is supported by the municipalities of Giove, Attigliano, Bastia Umbra, Penna in Teverina. In this way it is possible to identify the cause of a specific position for each municipality in the ranking.

The possibility of knowing how many rules are influenced by a certain indicator enables the contribution of each indicator in the final results to understand. Moreover, we can understand if a specific indicator has a positive, negative or neutral influence on the final results, and in how many cases. It is very important to know which characteristics are fundamental for achieving a good level of sustainable development.

Sustainability is a multidimensional concept; rural areas present very different environments, a variety of economic activities, social and cultural traditions. Thus, decision makers deal with a high level of complexity when involved in assessment and decision making activities in this field. The clear results given by DRSA allow DMs to address specific actions in specific regions. This type of analysis also enables to point out weak points of each municipality corresponding to the conditions of the $\mathrm{D} \leq$-decision rules classifying the municipalities as at most average or poor to be corrected, as well as to point out the good features of each municipality corresponding to the conditions of the $\mathrm{D} \geq$-decision rules classifying the municipalities as at least average or good to be strengthened. 


\section{Conclusions}

A dominance-based Rough Set Approach was applied to sustainable rural development evaluations. We showed that DRSA has many good properties within this context: transparency, the use of qualitative evaluations, decision analysis based on ordinal properties only, and traceability. The Decision maker gives preferential information by simply answering easy questions, and obtains transparent feedback in a learning oriented perspective. For this reason, the method can be considered as a "glass box" (Greco et al., 2001a). In addition, decision makers get good support from this approach, but of course they are "not forced" to follow the results and recommendations of the DRSA application. Instead, DRSA supplies clear and "open" explanations of the problem to be solved and more information for their decisions.

The methodology presented in this paper is also available in a complete MCDA-GIS integration system with four different MCDA modules: ELECTRE, Fuzzy set, Regime and DRSA. The MCDA suite is available in the GRASS add-ons repository (http://trac.osgeo.org/grass/browser/grassaddons/raster/mcda) with GNU GPL license.

With reference to the case study, rural sustainable development is a multidimensional concept. A huge quantity of data is needed for the description and evaluation of rural sustainable development, and this data must be processed using proper methodologies, capable of supporting the decision maker. DRSA is a suitable methodology in this context. However, further development is necessary for rural sustainable development assessment: the set of indicators must be improved both in number and in the type of information.

Future work could relate to the application of the DRSA for decisions involving a plurality of decision makers (Greco et al., 2006) (stakeholders such as Public Administration, Environmental associations, Trade unions, Enterprises, etc.). 


\section{References}

Ayres, W.S., McCalla, A.F., 1996. Rural development, agriculture and food security. Financ. Dev. 33, 8-11.

Baldock, D., Dwyer, J., Lowe, P., Petersen, J., Ward, N. 2001. The Nature of Rural development: towards a sustainable integrated rural policy in Europe. Synthesis Report, IEEP, London.

Blaszczynski, J., Greco, S., Slowinski, R., Szelag M., 2009. Monotonic variable consistency rough set approaches. Int. J. Approx. Reason. 50 (7), 979-999.

Copus, A.K., Crabtree, J.R., 1996. Indicators of Socio-economic Sustainability: an Application to Rural Remote Scotland, J. Rural Stud. 12, 41-54.

Emerson, H.J., Gillmor, D.A., 1999. The rural environment protection scheme of the Republic of Ireland. Land Use Policy 16, 235-245.

European Commission, 1997. Reform of the structural funds. COM (98) 131 def.-98/0090, 19 March.

FAO, 1988. Aspects of FAO's Policies, Programmes, Budget and Activities Contributing to Sustainable Development. Report of the Director General FAO, presented to the FAO Council, FAO, Rome.

Figueira, J., Greco, S. Ehrgott, M. (Eds.), 2005. Multiple Criteria Decision Analysis: State of the Art Surveys. Springer, New York.

Gómez-Limón, J.A., Atance, I., 2004. Identification of public objectives related to agricultural sector support. J. Policy Model. 26, 1045-1071.

Greco, S., Matarazzo, B., Slowinski, R., 1999. The use of rough sets and fuzzy sets in MCDM. In: Gal, T., Hanne, T., Stewart T. (Eds), Advances in Multiple Criteria Decision Making. Kluwer, Dordrecht.

Greco, S., Matarazzo, B., Slowinski, R., 2001a. Rough set theory for multi-criteria decision analysis. European Eur. J. Oper. Res. 129.

Greco, S., Matarazzo, B., Slowinski, R., 2002. Rough approximation by Dominance Relations, International Int. J. Intell. Syst. 17.

Greco, S., Matarazzo, B., Slowinski, R., 2005. Decision rule approach. Chapter 13. In: Figueira J., Greco S., Ehrgott M. (Eds.), Multiple Criteria Decision Analysis: State of the Art Surveys. Springer, New York, 507-562.

Greco, S., Matarazzo, B., Slowinski R., 2006. Dominance-Based Rough Set Approach to Decision Involving Multiple Decision Makers. In: Greco, S., Hata, Y., Hirano, S., Inuiguchi, M., Miyamoto, S., Nguyen, H.S., 
Slowinski, R. (Eds.), Rough Sets and Current Trends in Computing (RSCTC 2006). Springer LNCS 4259, Berlin, 306-317.

Greco, S., Matarazzo, B., Slowinski, R., 2008. Dominance-Based Rough Set Approach to Interactive Multiobjective Optimization. In: Branke J., Deb K., Miettinen K., Slowinski R. (Eds.), Multi-objective Optimization: Interactive and Evolutionary Approaches. Springer-Verlag, Berlin.

Greco, S., Matarazzo, B., Slowinski, R., Stefanowski, J., 2001b. Variable consistency model of dominancebased rough set approach. In: Ziarko W., Yao Y. (Eds.), Rough Sets and Current Trends in Computing. Springer LNAI 2005, Berlin, 170-181.

Hart, K., Baldock, D., 2011. Greening the CAP: delivering environmental outcomes through pillar one. IEEP, London.

Heilig, G.K., 2002. European Rural Development, International Institute for Applied Systems Analysis, Laxenburg. Austria.

Hjalager, A., 1999. Agriculture diversification into tourism: evidence of a European community development programme. Tourism Manage. 17, 103-111.

Istat, 2010. $6^{\circ}$ Agriculture Census. http://censimentoagricoltura.istat.it/

Istat, 2011. $15^{\circ}$ Population Census. http://censimentopopolazione.istat.it/

Jonard, F., Lambotte, M., Ramos, F., Terres, J.M., Bamps, C., 2009. Delimitations of rural areas in Europe using criteria of population density, remoteness and land cover. JRC Scientific Report, EUR 23757 EN.

Kaiser, B., 1990. La renaissance rurale. Armand Colin. Paris.

Kitchen, L., Marsden, T., 2009. Creating Sustainable rural development stimulating the eco-economy: beyond the eco-economic paradox? Sociol. Ruralis 49, 273-294.

Kneafsey, M., 2001. Rural economy: tourism and social relations. Annals of tourism research 28, 762-783.

Morgan, S.L., Marsden, T., Miele, M., Morley A., 2010. Agricultural multi-functionality and farmers' entrepreneurial skills: a study of Tuscan and Welsh farmers. J. Rural Stud. 26, 116-129.

Murdoch, J., Pratt, A.C., 1997. From the power of topography to the topography of power. A discourse on strange ruralities. In: Cloke, P., Little, J. (Eds.), Contested countryside cultures. Otherness, marginalisation and rurality. Routledge. London.

OECD, 1994. Creating rural indicators for shaping territorial policies. Oecd, Paris. 
OECD, 1996. Territorial indicators of employment - Focusing on rural development. Oecd, Paris.

Pawlak, Z., 1982. Rough sets. Int. J. Comput. Inf. Sci. 11.

Pawlak, Z., 1991. Rough Sets, Dordrecht, Kluwer.

Schultink, G., 2000. Critical environmental indicators: performance indices and assessment models for sustainable rural development planning. Ecol. Model. 130, 47-58.

Shortall, S., Shucksmith, M., 2001. Rural development in practice: issues arising in Scotland and Northern Ireland. Community Dev. J. 36, 122-133.

Slowinski, R., Greco, S., Matarazzo, B., 2009. Rough Sets in Decision Making. In: Meyers R.A. (Ed.), Encyclopedia of Complexity and Systems Science. Springer, New York, 7753-7786.

Slowinski, R., Greco, S., Matarazzo, B., 2012. Rough Set and Rule-based Multicriteria Decision Aiding. Pesqui. Oper. 32 (2), 213-269.

Slowinski, R., Greco, S., Matarazzo, B., 2014. Rough-Set-Based Decision Support. Chapter 19. In: Burke E.K., Kendall G. (Eds.), Search Methodologies: Introductory Tutorials in Optimization and Decision Support Techniques. 2nd edition, Springer, New York, 557-609.

Szlanyinka, E., 2009. The role of cultural values in rural development. In: de Noronha Vaz, T., Nijkamp, P., Rastoin, J. (Eds.), Traditional food production and rural sustainable development: a European challenge, Ashgate Publishing, Surrey.

United Nation, 2008. Urban and Rural Areas 2007. United Nation Publication Biodiversity and Conservation $14,1641-1661$.

Wilson, G.A., 2007. Multifunctional Agriculture: a transition Theory perspective. CABI, Wallinford.

Young, J., Watt, A., Nowick, P., Alard, D., Clitherow, J., Henle, K., Johnson, R., Laczko, E., Mccracken, D., Matouch, S., Niemela, J., Richards, C., 2005. Towards sustainable land use: identifying and managing the conflicts between human activities and biodiversity conservation in Europe. Biodivers. Conserv. 14, 16411661. 
Table 1 - A frame of the effects table

\begin{tabular}{|c|c|c|c|c|c|c|c|c|c|c|}
\hline Municipality & A1 & A2 & A3 & A4 & A5 & A6 & A7 & A8 & A9 & A10 \\
\hline 1. Acquasparta & 0.38 & 62.08 & 0.76 & 4,940 & 0.01 & 0.01 & 0.55 & 15.78 & 1.23 & 0.18 \\
\hline 2. Allerona & 0.43 & 22.66 & 0.82 & 1,863 & 0.01 & 0.02 & 2.76 & 19.22 & 0.69 & 0.00 \\
\hline 3. Alviano & 1.00 & 63.80 & 0.84 & 1,519 & 0.04 & 0.01 & 0.42 & 470.02 & 0.23 & 0.22 \\
\hline 4. Amelia & 0.39 & 89.11 & 0.80 & 11,811 & 0.01 & 0.01 & 3.56 & 0.00 & 5.98 & 0.15 \\
\hline 5. Arrone & 0.47 & 69.55 & 0.77 & 2,850 & 0.02 & 0.01 & 1.12 & 35.66 & 0.46 & 0.19 \\
\hline 6. Assisi & 0.29 & 146.95 & 0.76 & 27,456 & 0.01 & 0.02 & 8.33 & 35.50 & 0.73 & 0.08 \\
\hline 7. Attigliano & 0.07 & 184.11 & 0.72 & 1,924 & 0.09 & 0.01 & 0.00 & 361.34 & 1.73 & 0.12 \\
\hline 8. Avigliano & 0.40 & 50.21 & 0.79 & 2,577 & 0.02 & 0.01 & 1.15 & 1.30 & 0.25 & 0.05 \\
\hline 9. Baschi & 0.68 & 41.22 & 0.82 & 2,816 & 0.01 & 0.03 & 3.48 & 18.15 & 0.13 & 0.12 \\
\hline 10. Bastia Umbra & 0.07 & 785.08 & 0.77 & 21,684 & 0.04 & 0.01 & 1.99 & 157.42 & 1.78 & 0.03 \\
\hline 11. Bettona & 0.61 & 95.09 & 0.79 & 4,298 & 0.02 & 0.01 & 5.35 & 169.56 & 1.10 & 0.00 \\
\hline 12. Bevagna & 0.47 & 90.42 & 0.82 & 5,078 & 0.02 & 0.03 & 5.48 & 66.42 & 1.95 & 0.22 \\
\hline
\end{tabular}

Table 2 - Presence of criteria in decision rules

\begin{tabular}{|c|c|c|c|c|c|}
\hline & Good & $\geq$ Medium & $\leq$ Medium & Bad & Total \\
\hline A1: Population scattering index & 1 & 8 & 1 & 5 & 15 \\
\hline A2: Concentrated population/Sq. Km & 4 & 1 & 4 & 1 & 10 \\
\hline A3: Owned homes/Total homes & 11 & 18 & 18 & 9 & 56 \\
\hline A4: Total residents & 10 & 19 & 0 & 0 & 29 \\
\hline A5: Agricultural age structure & 7 & 1 & 13 & 1 & 22 \\
\hline A6: Typical products rate & 11 & 14 & 12 & 7 & 44 \\
\hline $\begin{array}{l}\text { A7: No. of bed spaces in rural } \\
\text { tourism accommodation/Sq. km }\end{array}$ & 6 & 17 & 15 & 16 & 54 \\
\hline A8: Irrigation water use/UAA & 13 & 14 & 14 & 12 & 53 \\
\hline A9: Livestock standard units/UAA & 7 & 25 & 8 & 4 & 44 \\
\hline A10: Organic UAA/UAA: it & 3 & 9 & 13 & 15 & 40 \\
\hline
\end{tabular}


Table 3 - Score and rules confidence of the first ten municipalities in the ranking

\begin{tabular}{|c|c|c|c|c|c|}
\hline Municipalities & Score & $\begin{array}{l}\text { Conf. Rule } \\
\text { Good }(a, b)\end{array}$ & $\begin{array}{l}\text { Conf. Rule } \\
\geq \text { medium }(a, b)\end{array}$ & $\begin{array}{l}\text { Conf. Rule } \\
\leq \text { medium }(a, b)\end{array}$ & $\begin{array}{l}\text { Conf. Rule } \\
\text { bad }(a, b)\end{array}$ \\
\hline Preci & 80.4625 & $1(29,26.72)$ & $1(61,56.03)$ & $0.79(3,2.28)$ & $\mathbf{0}(0,0)$ \\
\hline Lisciano Niccone & 63.8821 & $1(26,23.72)$ & $1(52,47.30)$ & $0.83(8,6.38)$ & $0.75(1,0.75)$ \\
\hline Sellano & 57.4499 & $1(21,21.72)$ & $1(49,44.35)$ & $0.86(10,7.86)$ & $0.75(1,0.75)$ \\
\hline Paciano & 56.8884 & $1(17,15.92)$ & $1(51,46.53)$ & $0.96(7,5.56)$ & $\mathbf{0}(0,0)$ \\
\hline Monteleone di Spoleto & 46.684 & $1(20,18.63)$ & $1(37,33.40)$ & $\mathbf{0 . 8 2}(5,3.85)$ & $0.75(2,1.5)$ \\
\hline Poggiodomo & 41.6369 & $1(17,15.63)$ & $1(39,35.44)$ & $0.88(11,8.68)$ & $0.75(1,0.75)$ \\
\hline Valtopina & 40.7967 & $1(15,13.30)$ & $1(51,46.13)$ & $1(18,16.03)$ & $1(3,2.61)$ \\
\hline Tuoro sul Trasimeno & 39.2205 & $1(9,7.97)$ & $1(50,45.29)$ & $1(16,14.04)$ & $\mathbf{0}(0,0)$ \\
\hline Nocera Umbra & 35.5808 & $1(9,7.72)$ & $1(48,43.39)$ & $1(16,13.91)$ & $0.86(2,1.61)$ \\
\hline Norcia & 33.0154 & $1(13,11.38)$ & $1(39,35.03)$ & $1(14,11.65)$ & $1(2,1.75)$ \\
\hline
\end{tabular}

a stands for number of rules, $b$ for sum of confidence 